Sādhanā Vol. 37, Part 3, June 2012, pp. 319-340. (c) Indian Academy of Sciences

\title{
A reduced polynomial chaos expansion method for the stochastic finite element analysis
}

\author{
B PASCUAL and S ADHIKARI*
}

School of Engineering, Swansea University, Singleton Park, Swansea SA2 8PP, UK e-mail: S.Adhikari@swansea.ac.uk

MS received 18 December 2010; revised 4 May 2011; accepted 13 December 2011

\begin{abstract}
The stochastic finite element analysis of elliptic type partial differential equations is considered. A reduced method of the spectral stochastic finite element method using polynomial chaos is proposed. The method is based on the spectral decomposition of the deterministic system matrix. The reduction is achieved by retaining only the dominant eigenvalues and eigenvectors. The response of the reduced system is expanded as a series of Hermite polynomials, and a Galerkin error minimization approach is applied to obtain the deterministic coefficients of the expansion. The moments and probability density function of the solution are obtained by a process similar to the classical spectral stochastic finite element method. The method is illustrated using three carefully selected numerical examples, namely, bending of a stochastic beam, flow through porous media with stochastic permeability and transverse bending of a plate with stochastic properties. The results obtained from the proposed method are compared with classical polynomial chaos and direct Monte Carlo simulation results.
\end{abstract}

Keywords. Stochastic differential equations; polynomial chaos; reduced methods; spectral decomposition; numerical methods.

\section{Introduction}

Due to the significant development in computational hardware it is now possible to solve high resolution models in various computational physics problems, ranging from fluid mechanics to nano-bio mechanics. However, the spatial resolution is not enough to determine the credibility of a numerical model. A correct representation of the physical model as well as its parameters is also crucial. Since neither of these may be exactly known, over the past three decades there has been increasing research activities to model the governing partial differential equations within the framework of stochastic equations. We refer to few recent review papers, for example by

*For correspondence 
Nouy (2009); Charmpis et al (2007); Stefanou (2009) for comprehensive details. Consider a bounded domain $\mathcal{D} \in \mathbb{R}^{d}$ with piece-wise Lipschitz boundary $\partial \mathcal{D}$, where $d \leq 3$ is the spatial dimension. Further, we define a probability space $(\Omega, \mathcal{F}, P)$, where $\omega \in \Omega$ is a sample point from the sampling space $\Omega, \mathcal{F}$ is the complete $\sigma$-algebra over the subsets of $\Omega$ and $P$ is the probability measure. We analyse the stochastic elliptic partial differential equation (PDE)

$$
-\nabla[a(\mathbf{x}, \omega) \nabla u(\mathbf{x}, \omega)]=p(\mathbf{x}) ; \quad \mathbf{x} \text { in } \mathcal{D}
$$

with the associated Dirichlet condition

$$
u(\mathbf{x}, \omega)=0 ; \quad \mathbf{x} \text { on } \partial \mathcal{D} .
$$

Here $a: \mathbb{R}^{d} \times \Omega \rightarrow \mathbb{R}$ is a random field (Vanmarcke 1983), which can be viewed as a set of random variables indexed by $\mathbf{x} \in \mathbb{R}^{d}$. We assume the random field $a(\mathbf{x}, \omega)$ to be stationary and square integrable. Depending on the physical problem, the random field $a(\mathbf{x}, \omega)$ can be used to model different physical quantities. As an example, for a slow flow of an incompressible, viscous fluid through a porous media, $a(\mathbf{x}, \omega)$ would be the random field describing the permeability of the medium. The purpose of this paper is to investigate a new solution approach for Eq. (1) after the discretization using the stochastic finite element method (Ghanem \& Spanos 1991; Kleiber \& Hien 1992; Matthies et al 1997; Manohar \& Adhikari 1998).

Among the various techniques to solve stochastic partial differential equations, spectral stochastic finite element methods (SSFEM) have received significant attention. Although this method has been applied to various practical problems, one of the possible drawbacks is the high computational cost associated with large systems. As a result, various reduction methods have been proposed over the past decade. The reduction of the system can be done before or after applying the spectral decomposition such as the polynomial chaos (PC) expansion. Here the response is expressed as a linear combination of a set of vectors where the coefficients are expanded with PC. For example, Sachdeva et al (2006) used the first basis vectors spanning the preconditioned stochastic Krylov subspace, and each vector was expressed as a linear combination of a deterministic vector and a PC. An orthogonalization of the deterministic response and its first-order derivative with respect to parameters and design parameters, calculated at calibration points was used by Maute et al (2009). A Galerkin based reduced spectral function approach was proposed by Adhikari (2011). A different condensation method focused on dynamic systems was developed by Guedri et al (2006), where the vectors used were a reduced basis, e.g., a Ritz basis of fixed or free normal modes, and a static displacement where the uncertainty in the dynamic stiffness matrix is taken into account. In Acharjee \& Zabaras (2006) the generalized PC was applied to the diffusion equation, and the vectors used were the ones from the proper orthogonal decomposition obtained from the underlying deterministic diffusion problem.

The method proposed in this paper is based on a space reduction of the original system combined with a polynomial chaos approach. In section 2 a brief overview of spectral stochastic finite element method (SSFEM) is presented. The reduction technique is developed in section 3. The post processing of the results to obtain the response moments, and an error estimation of the proposed method with respect to SSFEM are discussed in section 4. Based on the theoretical results, a simple computational approach is proposed in section 5 . The new approach is applied to the stochastic mechanics of a beam in section 6 , to a flow through a stochastic porous medium in section 7 and to the stochastic mechanics of a plate in section 8 . From the theoretical developments and numerical results, some conclusions are drawn in section 9. 


\section{Overview of the spectral stochastic finite element method}

\subsection{Discretization of the stochastic PDE}

Consider $a(\mathbf{x}, \omega)$ is a Gaussian random field with a covariance function $C_{a}: \mathbb{R}^{d} \times \mathbb{R}^{d} \rightarrow \mathbb{R}$ defined in the domain $\mathcal{D}$. Since the covariance function is square bounded, symmetric and positive definite, it can be represented by a spectral decomposition in an infinite dimensional Hilbert space. Using this spectral decomposition, the random process $a(\mathbf{x}, \omega)$ can be expressed (see for example, Ghanem \& Spanos (1991) and Papoulis \& Pillai (2002)) in a generalized series of Fourier type known as the Karhunen-Loève expansion

$$
a(\mathbf{x}, \omega)=a_{0}(\mathbf{x})+\sum_{i=1}^{\infty} \sqrt{\nu_{i}} \xi_{i}(\omega) \varphi_{i}(\mathbf{x}) .
$$

Here $a_{0}(\mathbf{x})$ is the mean function, $\xi_{i}(\omega)$ are uncorrelated standard Gaussian random variables, $v_{i}$ and $\varphi_{i}(\mathbf{x})$ are eigenvalues and eigenfunctions satisfying the integral equation $\int_{\mathcal{D}} C_{a}\left(\mathbf{x}_{1}, \mathbf{x}_{2}\right) \varphi_{j}\left(\mathbf{x}_{1}\right) \mathrm{d} \mathbf{x}_{1}=v_{j} \varphi_{j}\left(\mathbf{x}_{2}\right), \quad \forall j=1,2, \cdots$. Truncating the series (3) up to the $M$ th term, substituting $a(\mathbf{x}, \omega)$ in the governing PDE (1) and applying the boundary conditions, the discretized equation can be written as

$$
\left[\mathbf{A}_{0}+\sum_{i=1}^{M} \xi_{i}(\omega) \mathbf{A}_{i}\right] \mathbf{u}(\omega)=\mathbf{f} .
$$

The necessary technical details to obtain the discrete stochastic algebraic equations from the stochastic partial differential equation (1) has become standard in the literature and therefore omitted here. Excellent references, for example Ghanem \& Spanos (1991); Matthies \& Keese (2005) and Babuska et al (2005), are available on this topic. In Eq. (4) $\mathbf{A}_{0}$ is a symmetric positive definite matrix, $\mathbf{A}_{i} \in \mathbb{R}^{n \times n} ; i=1,2, \ldots, M$ are symmetric matrices, $\mathbf{u}(\omega) \in \mathbb{R}^{n}$ is the solution vector and $\mathbf{f} \in \mathbb{R}^{n}$ is the force vector. We assume that the eigenvalues of $\mathbf{A}_{0}$ are distinct. The number of terms $M$ in Eq. (4) can be selected based on the 'amount of information' to be retained. This in turn is related to the number of eigenvalues retained, since the eigenvalues, $v_{i}$, in Eq. (3) are arranged in a decreasing order. One of the main aims of a stochastic finite element analysis is to obtain $\mathbf{u}(\omega)$ for $\omega \in \Omega$ from Eq. (4) in an efficient manner and is the main topic of this paper.

\subsection{Brief review of the solution techniques}

The solution of the set of stochastic linear algebraic equations (4) is a key step in the stochastic finite element analysis. As a result, several methods have been proposed. These methods include, first- and second-order perturbation methods (Kleiber \& Hien 1992; Liu et al 1986; Adhikari 1999, 2000), Neumann expansion method (Yamazaki et al 1988; Adhikari \& Manohar 2000), Galerkin approach (Grigoriu 2006) and linear algebra based methods (Falsone \& Impollonia 2002; Li et al 2006; Feng 2007). More recently very efficient collocation methods have been proposed by Foo \& Karniadakis (2010) and Ma \& Zabaras (2009). Another class of methods which have been used widely in the literature are known as the spectral methods (see Nouy (2009) for a recent review). These methods include the polynomial chaos (PC) expansion (Ghanem \& Spanos 1991; Ghosh et al 2005), stochastic reduced basis method (Nair \& Keane 2002), and Wiener-Askey chaos expansion (Xiu \& Karniadakis 2002; Wan \& Karniadakis 2006) and 
reduced spectral function method (Adhikari 2011). According to the polynomial chaos expansion, second-order random variables $u_{j}(\omega)$ can be represented by the mean-square convergent expansion

$$
\begin{aligned}
u_{j}(\omega) & =u_{i_{0}}^{(j)} h_{0}+\sum_{i_{1}=1}^{\infty} u_{i_{1}}^{(j)} h_{1}\left(\xi_{i_{1}}(\omega)\right) \\
& +\sum_{i_{1}=1}^{\infty} \sum_{i_{2}=1}^{i_{1}} u_{i_{1}, i_{2}}^{(j)} h_{2}\left(\xi_{i_{1}}(\omega), \xi_{i_{2}}(\omega)\right)+\sum_{i_{1}=1}^{\infty} \sum_{i_{2}=1}^{i_{1}} \sum_{i_{3}=1}^{i_{2}} u_{i_{1} i_{2} i_{3}}^{(j)} h_{3}\left(\xi_{i_{1}}(\omega), \xi_{i_{2}}(\omega), \xi_{i_{3}}(\omega)\right) \\
& +\sum_{i_{1}=1}^{\infty} \sum_{i_{2}=1}^{i_{1}} \sum_{i_{3}=1}^{i_{2}} \sum_{i_{4}=1}^{i_{3}} u_{i_{1} i_{2} i_{3} i_{4}}^{(j)} h_{4}\left(\xi_{i_{1}}(\omega), \xi_{i_{2}}(\omega), \xi_{i_{3}}(\omega), \xi_{i_{4}}(\omega)\right)+\ldots,
\end{aligned}
$$

where $u_{i_{1}, \ldots, i_{p}}^{(j)}$ and $u_{i_{0}}^{(j)}$ are deterministic constants to be determined and $h_{p}\left(\xi_{i_{1}}(\omega), \ldots, \xi_{i_{p}}(\omega)\right)$ is the $p$ th order Homogeneous Chaos. When $\xi_{i}(\omega)$ are Gaussian random variables, the functions $h_{p}\left(\xi_{i_{1}}(\omega), \ldots, \xi_{i_{p}}(\omega)\right)$ are the $p$ th order hermite polynomial, defined as orthogonal with respect to the Gaussian probability density function. The same idea can be extended to non-Gaussian random variables, provided more generalized functional basis are used (Xiu \& Karniadakis 2002; Wan \& Karniadakis 2006). When we have a random vector, as in the case of the solution of Eq. (4), then it is natural to 'replace' the constants $u_{i_{1}, \ldots i_{p}}^{(j)}, u_{i_{0}}^{(j)}$ by vectors $\mathbf{u}_{i_{1}, \ldots i_{p}}^{(j)}, \mathbf{u}_{i_{0}}^{(j)} \in \mathbb{R}^{n}$. Suppose the series is truncated after $P$ number of terms.

Concisely, the polynomial chaos expansion for the solution of Eq. (4) can be written as

$$
\mathbf{u}(\omega)=\sum_{k=1}^{P} H_{k}(\boldsymbol{\xi}(\omega)) \mathbf{u}_{k},
$$

where $H_{k}(\boldsymbol{\xi}(\omega))$ are the polynomial chaoses. The unknown vectors $\mathbf{u}_{k}$ can be obtained by substituting $\mathbf{u}(\omega)$ from the above expressions into Eq. (4) and minimizing the error using a Galerkin approach (Ghanem \& Spanos 1991). It can be shown that we need to solve a $n P \times n P$ system of linear equations to obtain all $\mathbf{u}_{k} \in \mathbb{R}^{n}$ :

$$
\left[\begin{array}{ccc}
\mathbf{A}_{1,1} & \cdots & \mathbf{A}_{1, P} \\
\mathbf{A}_{2,1} & \cdots & \mathbf{A}_{2, P} \\
\vdots & \vdots & \vdots \\
\mathbf{A}_{P, 1} & \cdots & \mathbf{A}_{P, P}
\end{array}\right]\left\{\begin{array}{c}
\mathbf{u}_{1} \\
\mathbf{u}_{2} \\
\vdots \\
\mathbf{u}_{P}
\end{array}\right\}=\left\{\begin{array}{c}
\mathbf{f}_{1} \\
\mathbf{f}_{2} \\
\vdots \\
\mathbf{f}_{P}
\end{array}\right\} .
$$

The value of $P$ depends on the number of basic random variables $M$ and the order of the PC expansion $r$ as

$$
P=\sum_{j=0}^{r} \frac{(M+j-1) !}{j !(M-1) !} .
$$

Since $P$ increases very rapidly with the order of the chaos $r$ and the number of random variables $M$, the final number of unknown constants $P n$ becomes very large. As a result several methods have been developed (see for example Nair \& Keane (2002); Sarkar et al (2009) and Blatman \& Sudret (2010)) to reduce the computational cost. We investigate here the possibility of an alternative approach, where the system matrices are transformed into reduced dimensional matrices. 


\section{Development of the reduced polynomial chaos approach}

Following the spectral stochastic finite element method, an approximation to the solution of Eq. (4) can be expressed as a linear combination of functions of random variables and deterministic vectors. Recently Nouy $(2007,2008)$ discussed the possibility of an optimal spectral decomposition. The aim is to use small number of terms to reduce the computation time without loosing accuracy. A new approach is proposed here to reduce the dimension of the linear system of equation (7) arising in the PC method.

To illustrate the motivation behind the proposed reduced method, first consider the deterministic system

$$
\mathbf{A}_{0} \mathbf{u}_{0}=\mathbf{f}
$$

Because $\mathbf{A}_{0}$ is a symmetric and positive definite matrix, its eigenvalues are positive and its eigenvectors form a complete orthonormal basis. The eigenvalue problem can be expressed as

$$
\mathbf{A}_{0} \boldsymbol{\phi}_{k}=\lambda_{0_{k}} \boldsymbol{\phi}_{k} ; \quad k=1,2, \ldots n .
$$

For notational convenience, define the matrix of eigenvalues and eigenvectors

$$
\boldsymbol{\Lambda}_{0}=\operatorname{diag}\left[\lambda_{0_{1}}, \lambda_{0_{2}}, \ldots, \lambda_{0_{n}}\right] \in \mathbb{R}^{n \times n} \quad \text { and } \quad \boldsymbol{\Phi}=\left[\boldsymbol{\phi}_{1}, \boldsymbol{\phi}_{2}, \ldots, \boldsymbol{\phi}_{n}\right] \in \mathbb{R}^{n \times n} .
$$

Eigenvalues are ordered in the ascending order so that $\lambda_{0_{1}}<\lambda_{0_{2}}<\ldots<\lambda_{0_{n}}$. Since $\boldsymbol{\Phi}$ is an orthogonal matrix, we have $\boldsymbol{\Phi}^{-1}=\boldsymbol{\Phi}^{T}$ so that the following identities can be easily established

$$
\boldsymbol{\Phi}^{T} \mathbf{A}_{0} \boldsymbol{\Phi}=\Lambda_{0} ; \quad \mathbf{A}_{0}=\boldsymbol{\Phi}^{-T} \boldsymbol{\Lambda}_{0} \boldsymbol{\Phi}^{-1} \quad \text { and } \quad \mathbf{A}_{0}^{-1}=\boldsymbol{\Phi} \boldsymbol{\Lambda}_{0}^{-1} \boldsymbol{\Phi}^{T}
$$

These equations are also valid for the case where multiplicities in eigenvalues arise. Using these, the solution of Eq. (9) can be expressed as

$$
\mathbf{u}_{0}=\mathbf{A}_{0}^{-1} \mathbf{f}=\boldsymbol{\Phi} \boldsymbol{\Lambda}_{0}^{-1}\left(\boldsymbol{\Phi}^{T} \mathbf{f}\right)=\sum_{k=1}^{n} \frac{\boldsymbol{\phi}_{k}^{T} \mathbf{f}}{\lambda_{0_{k}}} \boldsymbol{\phi}_{k} .
$$

The series (13) can be truncated based on the magnitude of the eigenvalues as the higher terms becomes smaller. Therefore one could only retain the dominant terms in the series. If the system has an eigenvalue with multiplicities and small magnitude, all the series terms corresponding to this eigenvalue are retained in the truncated series. A similar model reduction technique has been widely used within the proper orthogonal decomposition method (Lenaerts et al 2002) where the eigenvalues of a symmetric positive definite matrix are used. One can select a small value $\epsilon$ such that $\lambda_{0_{1}} / \lambda_{0_{p}}<\epsilon$ for some value of $p$. Therefore, truncating the series in (13) one can have

$$
\mathbf{u}_{0} \approx \sum_{j=1}^{p} \frac{\boldsymbol{\phi}_{j}^{T} \mathbf{f}}{\lambda_{0_{j}}} \boldsymbol{\phi}_{j}
$$

We use this simple idea to develop the reduced PC (RPC) approach.

We form the reduced matrix of dominant eigenvalues and eigenvectors as

$$
\boldsymbol{\Lambda}_{0_{p}}=\operatorname{diag}\left[\lambda_{0_{1}}, \lambda_{0_{2}}, \ldots, \lambda_{0_{p}}\right] \in \mathbb{R}^{p \times p} \quad \text { and } \quad \boldsymbol{\Phi}_{p}=\left[\boldsymbol{\phi}_{1}, \boldsymbol{\phi}_{2}, \ldots, \boldsymbol{\phi}_{p}\right] \in \mathbb{R}^{n \times p} .
$$


Let us introduce the transformation

$$
\mathbf{u}(\omega)=\boldsymbol{\Phi}_{p} \mathbf{y}(\omega)
$$

where $\mathbf{y} \in \mathbb{R}^{p}$ is the new unknown random vector. Substituting in the original equation (4) and premultiplying by $\boldsymbol{\Phi}_{p}^{T}$ we have

$$
\left[\boldsymbol{\Lambda}_{0_{p}}+\sum_{i=1}^{M} \xi_{i}(\omega) \widetilde{\mathbf{A}}_{i}\right] \mathbf{y}(\omega)=\widetilde{\mathbf{f}},
$$

where the transformed reduced matrices and vector are given by

$$
\widetilde{\mathbf{A}}_{i}=\boldsymbol{\Phi}_{p}^{T} \mathbf{A}_{i} \boldsymbol{\Phi}_{p} \in \mathbb{R}^{p \times p} ; i=1,2, \ldots, M \quad \text { and } \quad \widetilde{\mathbf{f}}=\boldsymbol{\Phi}_{p}^{T} \mathbf{f}
$$

The main idea is to expand the reduced random vector $\mathbf{y}(\omega)$ with a polynomial chaos expansion. For a selected order of PC, the polynomial chaoses $H_{k}(\xi(\omega))$ will be identical to the ones used in the full system. Therefore, we have

$$
\mathbf{y}(\omega)=\sum_{k=1}^{P} H_{k}(\xi(\omega)) \mathbf{y}_{k} .
$$

The unknown vectors $\mathbf{y}_{k}$ can be obtained by substituting $\mathbf{y}(\omega)$ from the above expressions into the reduced Eq. (17) and minimizing the error using the standard Galerkin approach (Ghanem $\&$ Spanos 1991). It can be shown that we need to solve a $p P \times p P$ system of linear equation to obtain all $\mathbf{y}_{k} \in \mathbb{R}^{p}$ :

$$
\left[\begin{array}{ccc}
\widetilde{\mathbf{A}}_{1,1} & \cdots & \widetilde{\mathbf{A}}_{1, P} \\
\widetilde{\mathbf{A}}_{2,1} & \cdots & \widetilde{\mathbf{A}}_{1, P} \\
\vdots & \vdots & \vdots \\
\widetilde{\mathbf{A}}_{P, 1} & \cdots & \widetilde{\mathbf{A}}_{P, P}
\end{array}\right]\left\{\begin{array}{c}
\mathbf{y}_{1} \\
\mathbf{y}_{2} \\
\vdots \\
\mathbf{y}_{P}
\end{array}\right\}=\left\{\begin{array}{c}
\widetilde{\mathbf{f}}_{1} \\
\widetilde{\mathbf{f}}_{2} \\
\vdots \\
\widetilde{\mathbf{f}}_{P}
\end{array}\right\} .
$$

The computational complexity of the matrix inversion problem scales in cubically with the dimension of the matrix in the worse case (Wilkinson 1988). For many practical problems $p \ll n$, therefore $\mathcal{O}\left(P^{3} p^{3}\right) \ll \mathcal{O}\left(P^{3} n^{3}\right)$. As a result the RPC can offer significant computational reduction. This will be discussed further in the numerical examples later in the paper.

\section{Moments of the response and error analysis}

The mean and the covariance of the reduced system can be obtained using the standard PC approach. Suppose $\overline{\boldsymbol{y}}=\mathrm{E}[\mathbf{y}(\omega)]$. The covariance matrix of $\mathbf{y}(\omega)$ can be obtained using the orthogonality property of the polynomial chaoses as

$$
\boldsymbol{\Sigma}_{y}=\left(\sum_{k=1}^{P} \mathrm{E}\left[H_{k}^{2}(\boldsymbol{\xi}(\omega))\right] \mathbf{y}_{k} \mathbf{y}_{k}^{T}\right)-\mathbf{y}_{1} \mathbf{y}_{1}^{T} .
$$

The expressions of $\mathrm{E}\left[H_{k}^{2}(\xi(\omega))\right]$ can be obtained, for example from Ghanem \& Spanos (1991). Note that these calculations take less time than calculating the moments of the response using 
full PC, as the dimension of $\mathbf{y}_{k}$ is in general much smaller than the dimension of $\mathbf{u}_{k}$. The mean and the covariance matrix of the response of the original system can be obtained as

$$
\overline{\mathbf{u}}=\boldsymbol{\Phi}_{p} \overline{\mathbf{y}} \quad \text { and } \quad \boldsymbol{\Sigma}_{u}=\boldsymbol{\Phi}_{p} \boldsymbol{\Sigma}_{y} \boldsymbol{\Phi}_{p}^{T}
$$

The probability density function of $\mathbf{y}$ can be obtained by simulating Eq. (19). The samples of $\mathbf{u}$ can be generated easily using the samples of $\mathbf{y}$. To estimate the error between full PC and the proposed RPC, two different error analyses are given to try to evaluate the effect of neglecting the projection of the response on the eigenvectors not included in the new basis, that is, the eigenvectors corresponding to the largest eigenvalues. The first error estimate is based on the Schur complement, and gives an exact relationship between the approximation method and the full PC response. The second one is an heuristic approximation of the error, to understand the physical meaning of the neglected terms. The error is expected to be small when the projection of the response in the sub-space spanned by the deterministic eigenvectors corresponding to the higher eigenvalues is relatively smaller.

\subsection{Rigorous error analysis}

If the PC method is applied to the original system where all the KL expansion matrices have been premultiplied by $\boldsymbol{\Phi}^{T}$, the change of variables $\mathbf{u}=\boldsymbol{\Phi} \mathbf{y}^{*}$ is introduced and $\mathbf{f}^{*}=\boldsymbol{\Phi}^{T} \mathbf{f}$, the following system of equations is obtained

$$
\left(\mathrm{E}\left[\boldsymbol{\Gamma}^{2}\right] \otimes \boldsymbol{\Lambda}_{0}+\sum_{i=1}^{M} \mathbf{c}_{i} \otimes \boldsymbol{\Phi}^{T} \mathbf{A}_{i} \boldsymbol{\Phi}\right)\left\{\begin{array}{c}
\mathbf{y}_{1}^{*} \\
\vdots \\
\mathbf{y}_{P}^{*}
\end{array}\right\}=\left\{\begin{array}{c}
\mathbf{f}_{1}^{*} \\
\vdots \\
\mathbf{f}_{P}^{*}
\end{array}\right\} .
$$

The $k$ th diagonal entry of the diagonal matrix $\mathrm{E}\left[\Gamma^{2}\right]$ is $\mathrm{E}\left[\Gamma_{k}^{2}\right]$, where $\Gamma_{k}$ is the $k$ th Hermite polynomial used in the expansion. The $j k$ terms of matrices $\mathbf{c}_{i}$ are given by $c_{i j k}=\mathrm{E}\left[\xi_{i} \Gamma_{j} \Gamma_{k}\right]$. The terms of the SSFEM deterministic matrix can be rearranged such that the matrix can be partitioned as follows

$$
\left\{\begin{array}{ll}
\mathbf{B}_{11} & \mathbf{B}_{12} \\
\mathbf{B}_{21} & \mathbf{B}_{22}
\end{array}\right\}\left\{\begin{array}{c}
\mathbf{y}^{p *} \\
\mathbf{y}^{2 *}
\end{array}\right\}=\left\{\begin{array}{c}
\mathbf{f}^{p *} \\
\mathbf{f}^{2 *}
\end{array}\right\} \text {, with }\left\{\begin{array}{l}
\mathbf{y}^{p *} \\
\mathbf{y}^{2 *}
\end{array}\right\}=\left\{\begin{array}{c}
\mathbf{y}_{1_{p}}^{*} \\
\vdots \\
\mathbf{y}_{P_{p}}^{*} \\
\mathbf{y}_{1_{2}}^{*} \\
\vdots \\
\mathbf{y}_{P_{2}}^{*}
\end{array}\right\} \text { and }\left\{\begin{array}{l}
\mathbf{f}^{p *} \\
\mathbf{f}^{2 *}
\end{array}\right\}=\left\{\begin{array}{c}
\tilde{\mathbf{f}}_{1} \\
\vdots \\
\tilde{\mathbf{f}}_{P} \\
\mathbf{f}_{1_{2}}^{*} \\
\vdots \\
\mathbf{f}_{P_{2}}^{*}
\end{array}\right\} .
$$

The submatrices are given by

$$
\begin{array}{ll}
\mathbf{B}_{11}=\mathrm{E}\left[\boldsymbol{\Gamma}^{2}\right] \otimes \boldsymbol{\Lambda}_{0_{p}}+\sum_{1}^{M} \mathbf{c}_{i} \otimes \widetilde{\mathbf{A}}_{i}, & \mathbf{B}_{12}=\sum_{1}^{M} \mathbf{c}_{i} \otimes \boldsymbol{\Phi}_{p}^{T} \mathbf{A}_{i} \boldsymbol{\Phi}_{2} . \\
\mathbf{B}_{21}=\sum_{1}^{M} \mathbf{c}_{i} \otimes \boldsymbol{\Phi}_{2}^{T} \mathbf{A}_{i} \boldsymbol{\Phi}_{p} \quad \text { and } & \mathbf{B}_{22}=\mathrm{E}\left[\boldsymbol{\Gamma}^{2}\right] \otimes \boldsymbol{\Lambda}_{0_{2}}+\sum_{1}^{M} \mathbf{c}_{i} \otimes \boldsymbol{\Phi}_{2}^{T} \mathbf{A}_{i} \boldsymbol{\Phi}_{2} .
\end{array}
$$


The vectors appearing in (23) have been partitioned and rearranged in new vectors such that

$$
\mathbf{y}_{j}^{*}=\left\{\begin{array}{c}
\mathbf{y}_{j_{p}}^{*} \\
\mathbf{y}_{j_{2}}^{*}
\end{array}\right\} \text {, with } \mathbf{y}^{p *}=\left\{\begin{array}{c}
\mathbf{y}_{1_{p}}^{*} \\
\vdots \\
\mathbf{y}_{P_{p}}^{*}
\end{array}\right\} \text { and } \mathbf{y}^{2 *}=\left\{\begin{array}{c}
\mathbf{y}_{1_{2}}^{*} \\
\vdots \\
\mathbf{y}_{P_{2}}^{*}
\end{array}\right\} .
$$

The forcing term from (23) is related to the one in (24) through

$$
\mathbf{f}_{k}^{*}=\left\{\begin{array}{c}
\tilde{\mathbf{f}}_{k} \\
\mathbf{f}_{k_{2}}^{*}
\end{array}\right\}, \text { with } \mathbf{f}^{p *}=\left\{\begin{array}{c}
\tilde{\mathbf{f}}_{1} \\
\vdots \\
\tilde{\mathbf{f}}_{P}
\end{array}\right\} \quad \text { and } \quad \mathbf{f}^{2 *}=\left\{\begin{array}{c}
\mathbf{f}_{1_{2}}^{*} \\
\vdots \\
\mathbf{f}_{P_{2}}^{*}
\end{array}\right\} .
$$

Making use of the Schur complement (see, for example, Zhang (2005)), the partitioned solution can be written as

$$
\left\{\begin{array}{l}
\mathbf{y}^{p *} \\
\mathbf{y}^{2 *}
\end{array}\right\}=\left\{\begin{array}{l}
\left(\mathbf{B}_{11}-\mathbf{B}_{12} \mathbf{B}_{22}^{-1} \mathbf{B}_{21}\right)^{-1}\left(\mathbf{f}^{p *}-\mathbf{B}_{12} \mathbf{B}_{22}^{-1} \mathbf{f}^{2 *}\right) \\
\left(\mathbf{B}_{22}-\mathbf{B}_{21} \mathbf{B}_{11}^{-1} \mathbf{B}_{12}\right)^{-1}\left(\mathbf{f}^{2 *}-\mathbf{B}_{21} \mathbf{B}_{11}^{-1} \mathbf{f}^{p *}\right)
\end{array}\right\} .
$$

The response calculated by the proposed reduced method is given by $\mathbf{y}_{p}=\mathbf{B}_{11}^{-1} \mathbf{f}^{p *}$. As it can be observed, in the proposed method it is assumed that the non-diagonal block matrices are zero and that higher eigenvalues or the uncertainty linked to them have no effect on the response. The error associated to the proposed method with respect to the full system is given by

$$
\varepsilon=\left(\mathbf{u}^{*}-\mathbf{u}\right)^{T}\left(\mathbf{u}^{*}-\mathbf{u}\right), \quad\left(\mathbf{u}^{*}-\mathbf{u}\right)=\left(\boldsymbol{\Phi}\left[\begin{array}{c}
\mathbf{y}_{p}^{*} \\
\mathbf{y}_{2}^{*}
\end{array}\right]-\boldsymbol{\Phi}\left[\begin{array}{c}
\mathbf{y}_{p} \\
0
\end{array}\right]\right)=\boldsymbol{\Phi}\left(\left[\begin{array}{c}
\mathbf{y}^{p *}-\mathbf{y}_{p} \\
\mathbf{y}^{2 *}
\end{array}\right]\right) .
$$

An approximation can be introduced to the expression of $\mathbf{y}^{p *}$ using the Neumann expansion

$$
\mathbf{y}^{p *} \approx\left(\sum_{s=0}^{\infty}\left(\mathbf{B}_{11}^{-1} \mathbf{B}_{12} \mathbf{B}_{22}^{-1} \mathbf{B}_{21}\right)^{s}\right) \mathbf{B}_{11}^{-1}\left(\mathbf{f}^{p *}-\mathbf{B}_{12} \mathbf{B}_{22}^{-1} \mathbf{f}^{2 *}\right)
$$

and we obtain

$$
\mathbf{y}^{p *}-\mathbf{y}^{p} \approx\left(\sum_{s=1}^{\infty}\left(\mathbf{B}_{11}^{-1} \mathbf{B}_{12} \mathbf{B}_{22}^{-1} \mathbf{B}_{21}\right)^{s}\right) \mathbf{B}_{11}^{-1}\left(\mathbf{f}^{p *}-\mathbf{B}_{12} \mathbf{B}_{22}^{-1} \mathbf{f}^{2 *}\right)+\mathbf{B}_{11}^{-1}\left(-\mathbf{B}_{12} \mathbf{B}_{22}^{-1} \mathbf{f}^{2 *}\right) .
$$

These expressions, although correct, do not allow to grasp the physical meaning of neglecting the projection of the response on eigenvectors corresponding to the higher eigenvalues. The following approximate error analysis is given to fulfill this requirement.

\subsection{Approximate error analysis}

We consider an approximate error analysis to get an idea of the nature of the approximations involved in the proposed RPC. Let us partition the matrices of the eigenvalues and eigenvectors as

$$
\boldsymbol{\Lambda}_{0}=\left[\boldsymbol{\Lambda}_{0_{p}} \mid \boldsymbol{\Lambda}_{0_{2}}\right] \quad \text { and } \boldsymbol{\Phi}=\left[\boldsymbol{\Phi}_{p} \mid \boldsymbol{\Phi}_{2}\right] .
$$


Here the matrices $\boldsymbol{\Lambda}_{0_{2}} \in \mathbb{R}^{(n-p) \times(n-p)}$ and $\boldsymbol{\Phi}_{2} \in \mathbb{R}^{n \times(n-p)}$ denote the blocks that have not been used in the reduced method. Following the analogy of Eq. (13) and using the partitions in Eq. (33), the response can be expressed as

$$
\begin{aligned}
\mathbf{u}_{0}=\left[\boldsymbol{\Phi} \Lambda_{0}^{-1} \boldsymbol{\Phi}^{T}\right] \mathbf{f} & =\left(\left[\boldsymbol{\Phi}_{p} \mid \boldsymbol{\Phi}_{2}\right]\left[\boldsymbol{\Lambda}_{0_{p}} \mid \boldsymbol{\Lambda}_{0_{2}}\right]^{-1}\left[\boldsymbol{\Phi}_{p} \mid \boldsymbol{\Phi}_{2}\right]^{T}\right) \mathbf{f} \\
& =\left[\boldsymbol{\Phi}_{p} \boldsymbol{\Lambda}_{0_{p}}^{-1} \boldsymbol{\Phi}_{p}^{T}\right] \mathbf{f}+\left[\boldsymbol{\Phi}_{2} \boldsymbol{\Lambda}_{0_{2}}^{-1} \boldsymbol{\Phi}_{2}^{T}\right] \mathbf{f}=\boldsymbol{\Phi}_{p} \mathbf{y}_{0}+\left[\boldsymbol{\Phi}_{2} \boldsymbol{\Lambda}_{0_{2}}^{-1} \boldsymbol{\Phi}_{2}^{T}\right] \mathbf{f},
\end{aligned}
$$

where $\mathbf{y}_{0}=\Lambda_{0_{P}}^{-1} \boldsymbol{\Phi}_{P}^{T} \mathbf{f}$. Clearly in the proposed reduced approach when only $\mathbf{y}_{0}$ is considered, the second term is neglected.

Suppose, the error associated with the PC expansion of $\mathbf{u}(\omega)$ and $\mathbf{y}(\omega)$ are respectively $\boldsymbol{\varepsilon}_{u}$ and $\boldsymbol{\varepsilon}_{y}$. The additional error arising in the evaluation of $\mathbf{y}(\omega)$ comes from neglecting the term similar to the second term of (34), given by

$$
\boldsymbol{\varepsilon}_{2}=\left[\boldsymbol{\Phi}_{2} \boldsymbol{\Lambda}_{0_{2}}^{-1} \boldsymbol{\Phi}_{2}^{T}\right] \mathbf{f}
$$

Taking the $l_{2}$ norm we have

$$
\left\|\boldsymbol{\varepsilon}_{2}\right\|=\operatorname{Trace}\left(\left(\left[\boldsymbol{\Phi}_{2} \boldsymbol{\Lambda}_{0_{2}}^{-1} \boldsymbol{\Phi}_{2}^{T}\right] \mathbf{f}\right)\left(\left[\boldsymbol{\Phi}_{2} \boldsymbol{\Lambda}_{0_{2}}^{-1} \boldsymbol{\Phi}_{2}^{T}\right] \mathbf{f}\right)^{T}\right)=\operatorname{Trace}\left(\boldsymbol{\Phi}_{2}^{T} \boldsymbol{\Phi}_{2} \boldsymbol{\Lambda}_{0_{2}}^{-1} \boldsymbol{\Phi}_{2}^{T} \mathbf{f f}^{T} \boldsymbol{\Phi}_{2} \boldsymbol{\Lambda}_{0_{2}}^{-1}\right) .
$$

Recalling that $\boldsymbol{\Phi}_{2}^{T} \boldsymbol{\Phi}_{2}$ is an identity matrix we have

$$
\left\|\varepsilon_{2}\right\|=\operatorname{Trace}\left(\boldsymbol{\Lambda}_{0_{2}}^{-2} \boldsymbol{\Phi}_{2}^{T} \mathbf{f f}^{T} \boldsymbol{\Phi}_{2}\right) .
$$

Since $\boldsymbol{\Phi}_{p}$ is an orthonormal matrix, the error norm is invariant with respect to rotation in $\boldsymbol{\Phi}_{p}$. That is the error norm for $\mathbf{y}(\omega)$ and $\boldsymbol{\Phi}_{p} \mathbf{y}(\omega)$ are identical. Therefore, from Eq. (34) the error norm of $\mathbf{y}(\omega)$ and $\mathbf{u}(\omega)$ can be approximately related as

$$
\left\|\boldsymbol{\varepsilon}_{u}\right\| \gtrsim\left\|\boldsymbol{\varepsilon}_{y}\right\|+\operatorname{Trace}\left(\boldsymbol{\Lambda}_{0_{2}}^{-2} \boldsymbol{\Phi}_{2} \mathbf{f f}^{T} \boldsymbol{\Phi}_{2}^{T}\right) .
$$

This expression shows that the difference between the error norm of $\mathbf{u}(\omega)$ and $\mathbf{y}(\omega)$ will be small when the eigenvalues in the diagonal matrix $\boldsymbol{\Lambda}_{0_{2}}$ are large.

\section{Summary of the computational method}

The reduced polynomial chaos (RPC) for the solution of the stochastic elliptic PDE (1) can be implemented as follows:

(i) Obtain the system matrices $\mathbf{A}_{i}, i=0,1,2, \ldots, M$ and the forcing vector $\mathbf{f}$ by discretizing the governing stochastic partial differential equation using the well-established stochastic finite element methodologies.

(ii) Solve the eigenvalue problem associated with the mean matrix $\mathbf{A}_{0}$

$$
\mathbf{A}_{0} \Phi=\Lambda_{0} \Phi
$$

(iii) Select a small value of $\epsilon$, say $\epsilon=10^{-3}$. Obtain the number of the reduced orthonormal basis $p$ such that $\lambda_{0_{1}} / \lambda_{0_{p}}<\epsilon$. 
(iv) Create the reduced matrix of eigenvalues and eigenvectors

$$
\boldsymbol{\Lambda}_{0_{p}}=\operatorname{diag}\left[\lambda_{0_{1}}, \lambda_{0_{2}}, \ldots, \lambda_{0_{p}}\right] \in \mathbb{R}^{p \times p} \quad \text { and } \quad \boldsymbol{\Phi}_{p}=\left[\boldsymbol{\phi}_{1}, \boldsymbol{\phi}_{2}, \ldots, \boldsymbol{\phi}_{p}\right] \in \mathbb{R}^{n \times p} .
$$

(v) Calculate the transformed matrices and vector

$$
\widetilde{\mathbf{A}}_{i}=\boldsymbol{\Phi}_{p}^{T} \mathbf{A}_{i} \boldsymbol{\Phi}_{p} \in \mathbb{R}^{p \times p} ; i=1,2, \ldots, M \quad \text { and } \quad \widetilde{\mathbf{f}}=\boldsymbol{\Phi}_{p}^{T} \mathbf{f}
$$

(vi) Obtain the reduced polynomial chaos expansion

$$
\mathbf{y}(\omega)=\sum_{k=1}^{P} H_{k}(\boldsymbol{\xi}(\omega)) \mathbf{y}_{k},
$$

where the vectors $\mathbf{y}_{k} \in \mathbb{R}^{p}$ can be obtained by solving the $p P \times p P$ system of linear equation:

$$
\left[\begin{array}{ccc}
\widetilde{\mathbf{A}}_{1,1} & \cdots & \widetilde{\mathbf{A}}_{1, P} \\
\widetilde{\mathbf{A}}_{2,1} & \cdots & \widetilde{\mathbf{A}}_{2, P} \\
\vdots & \vdots & \vdots \\
\widetilde{\mathbf{A}}_{P, 1} & \cdots & \widetilde{\mathbf{A}}_{P, P}
\end{array}\right]\left\{\begin{array}{c}
\mathbf{y}_{1} \\
\mathbf{y}_{2} \\
\vdots \\
\mathbf{y}_{P}
\end{array}\right\}=\left\{\begin{array}{c}
\widetilde{\mathbf{f}}_{1} \\
\widetilde{\mathbf{f}}_{2} \\
\vdots \\
\tilde{\mathbf{f}}_{P}
\end{array}\right\} .
$$

(vii) Calculate the mean and the covariance matrix of the response of the original system

$$
\overline{\mathbf{u}}=\boldsymbol{\Phi}_{p} \overline{\mathbf{y}} \quad \text { and } \quad \boldsymbol{\Sigma}_{u}=\boldsymbol{\Phi}_{p} \boldsymbol{\Sigma}_{y} \boldsymbol{\Phi}_{p}^{T},
$$

where

$$
\boldsymbol{\Sigma}_{y}=\left(\sum_{k=1}^{P} \mathrm{E}\left[H_{k}^{2}(\boldsymbol{\xi}(\omega))\right] \mathbf{y}_{k} \mathbf{y}_{k}^{T}\right)-\mathbf{y}_{1} \mathbf{y}_{1}^{T} .
$$

The proposed RPC method is now applied to three numerical examples to verify the efficiency of the method.

\section{Transversal deformation of a beam with stochastic properties}

The method described is applied to the problem of a bending beam with stochastic flexural rigidity. We consider the numerical example of the clamped-free beam shown in figure 1, subjected to a deterministic transversal load at its tip of $P=0.4658 \mathrm{~N}$. The system is such that the finite element model of the beam has 50 elements and the associated deterministic matrix is of size $n=100$. The length of the Euler Bernoulli beam is $1 \mathrm{~m}$, and for the deterministic case, Young's modulus is $E=6910^{9} \mathrm{~N} / \mathrm{m}^{2}$ and the cross-section is a rectangle of length $40 \mathrm{~mm}$ and height

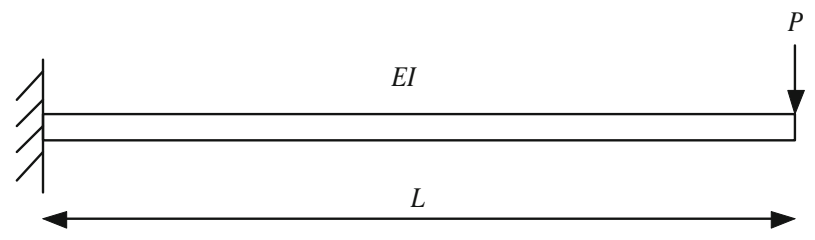

Figure 1. A clamped-free beam with random bending rigidity subjected to a point load at the end. 


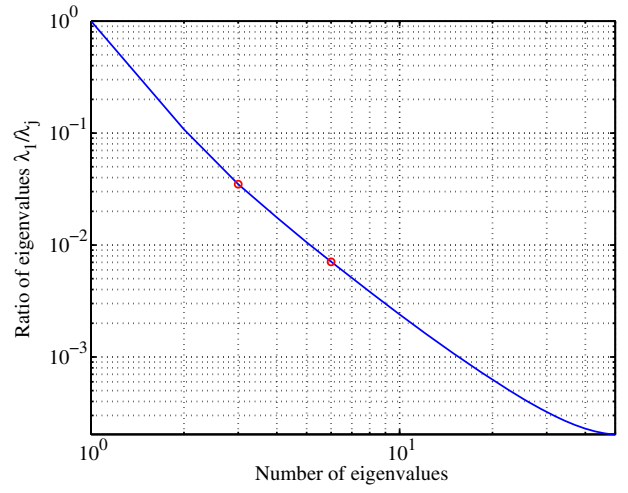

(a) Ratio of eigenvalues

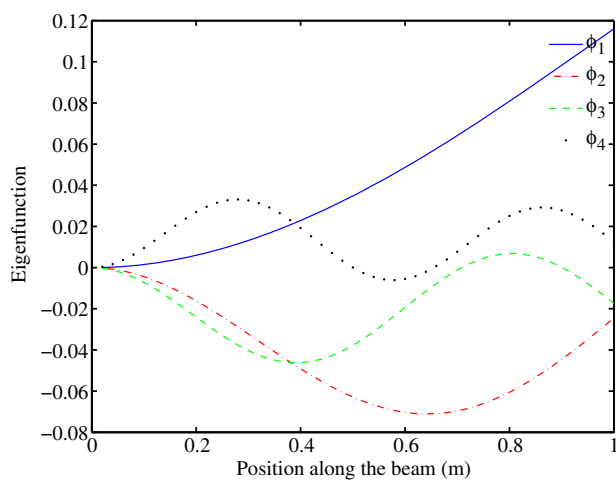

(b) Eigenvectors

Figure 2. Ratio of eigenvalues and eigenvectors for the beam problem.

$4 \mathrm{~mm}$, its moment of inertia is $I=910^{-11} \mathrm{~m}^{4}$. The flexural rigidity of the beam, $E I$, is considered as a random field with mean $\overline{E I}=6.21 \mathrm{Nm}^{2}$, standard deviation $0.2 \overline{E I}$ and exponential covariance function with correlation length the length of the beam. Two terms are kept in the KL expansion of the system stiffness matrix. The order of the Polynomial Chaos used is four, that is, 15 polynomials are used in the expansion of the response.

The solution is obtained with different methods to compare their performance. MCS method is applied using 10000 samples. The PC method, called here full PC, is applied, and the linear system to be solved is of size 1500. Two reduced systems are considered, using the first 3 and 6 eigenvectors. The ratio between the first and the $n$th eigenvalue is shown in figure 2, together with the first four eigenvectors for the transverse displacement. The two reduced PC system matrices are of sizes 45 and 90 for transformed stiffness matrices of sizes 3 and 6, respectively. Values of the mean and standard deviation of the vertical displacement for all the nodes are shown in figure 3 . The displacement is normalized by $P L^{3} / 3 E I$ such that the deterministic

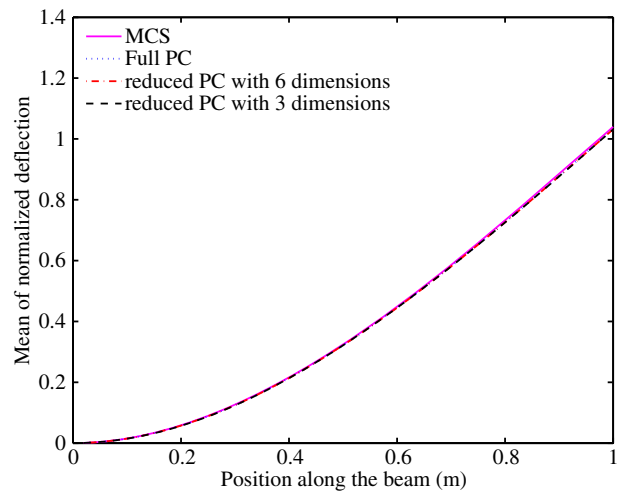

(a) Mean

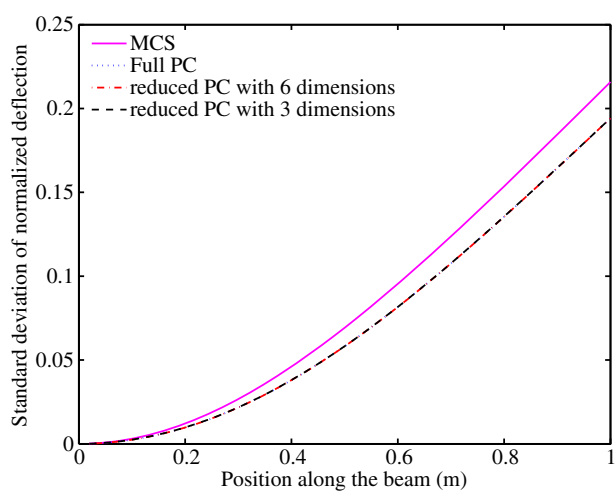

(b) Standard deviation

Figure 3. Mean and standard deviation of the normalized vertical displacement for the beam problem. 


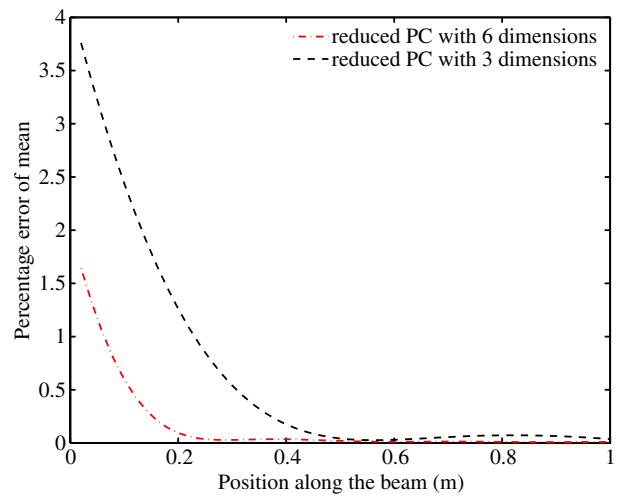

(a) Percentage error of mean

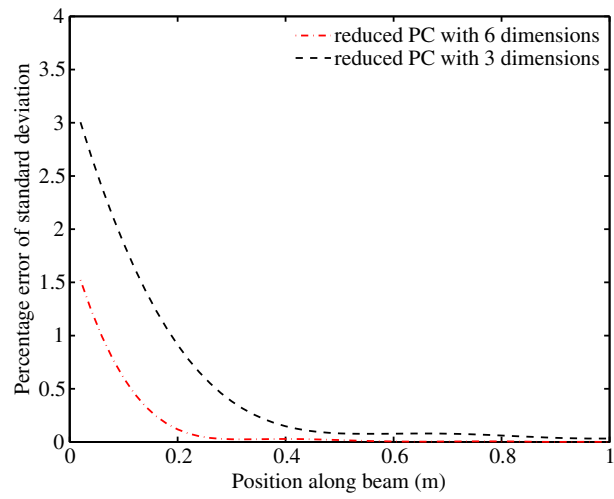

(b) Percentage error of standard deviation

Figure 4. Percentage error of mean and standard deviation of the normalized vertical displacement, between full PC and reduced PC.

vertical displacement at the tip is unity. The error associated with using RPC instead of the full $\mathrm{PC}$ is shown in figure 4 for all the nodes of the beam. The percentage error is represented as

$$
\varepsilon \%=100 \times \frac{|\mathrm{fPC}-\mathrm{RPC}|}{\mathrm{fPC}},
$$

where fPC are the results from full PC and RPC are the ones from the reduced PC. It is observed that the error is higher at position $x=0 \mathrm{~m}$, as values near the origin are close to zero. It is also observed that errors at the tip of the beam are negligible. As expected, the proposed method generates a response with an accuracy similar to the one of full PC, but at lower cost. This cost is described in table 1, where the CPU time of calculations for the four methods is given. Figure 5a shows the pdf of the tip vertical displacement for MCS, full PC and the two reduced systems. The error of the vertical displacement associated with using a reduced system instead of the full PC for the tip node is shown in figure $5 b$, for different orders of the reduced system. One can observe that with 6 dimensions, the proposed RPC produce results are comparable with the full PC with dimension 100. Figure 5b shows that the error compared to the full PC reduces significantly when the size of the reduced system is beyond 10 .

Table 1. CPU time (s) of calculations for the full PC and in the proposed reduced method. The cost of calculating complete eigensolutions is $0.0073 \mathrm{~s}$.

\begin{tabular}{ccccc}
\hline & MCS & Full PC $(n=100)$ & RPC $(n=3)$ & $\operatorname{RPC}(n=6)$ \\
\hline CPU time (s) & 1.1508 & 0.0238 & 0.0063 & 0.0071 \\
\hline
\end{tabular}




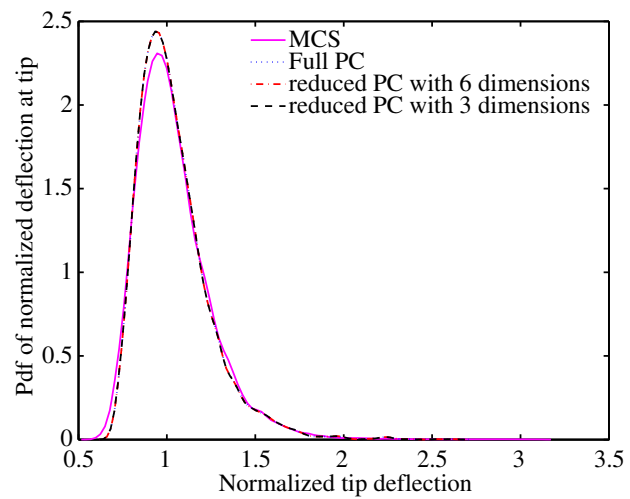

(a) Pdf of the normalized tip displacement.

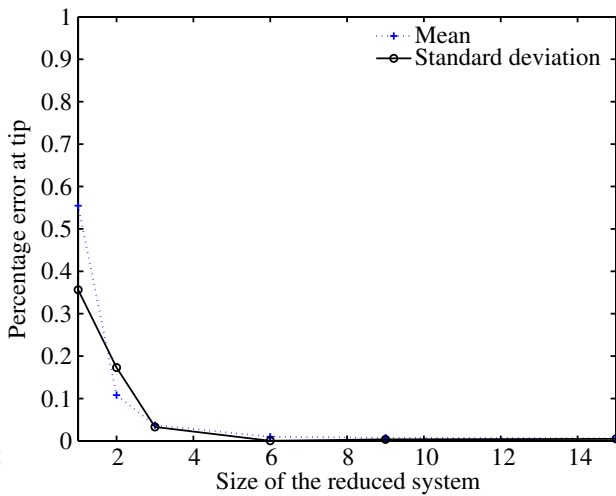

(b) Percentage errors of mean and standard deviation.

Figure 5. The pdf of the normalized vertical displacement at tip of the beam obtained with MCS, full PC and reduced PC with $n=3$ and $n=6$. Percentage errors of mean and standard deviation of the normalized tip displacement using different reduced systems.

\section{Flow through a stochastic medium}

A numerical example of flow through a porous media is now considered to investigate possible efficiency of the proposed method for higher spatial dimensional problem compared to the previous example. The two-dimensional domain is a rectangle of length $L=1 \mathrm{~m}$ and width $W=$ $0.6 \mathrm{~m}$, as shown in figure 6 . The domain is divided with a uniform mesh of 70 by 42 square elements. The domain is subjected to a constant flux $q_{b}=1 \mathrm{~cm} / \mathrm{s}$ along the portion of its boundary

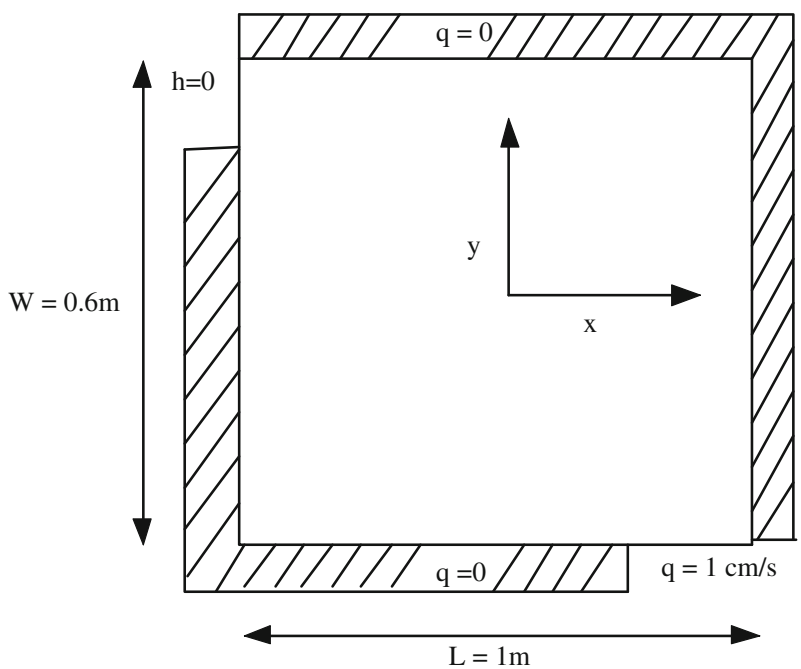

Figure 6. Flow through a rectangular porous media. The porous media is assumed to have stochastically inhomogeneous hydraulic conductivity. 


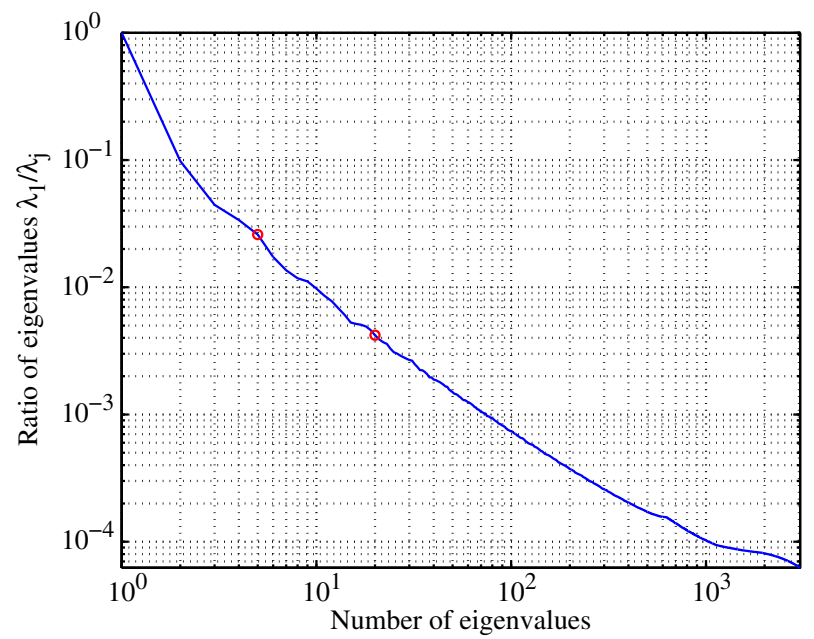

Figure 7. Ratio of eigenvalues for the flow through porous media problem.

verifying $y=-0.3 \mathrm{~m}$ and $x \in[0.3,0.5] \mathrm{m}$ (last 14 elements on $x$ direction), in coordinates of the KL expansion. The head is fixed at value $h_{b}=0 \mathrm{~cm}$ along the portion of the boundary such that $x=-0.5 \mathrm{~m}, y \in[0.1714,0.3] \mathrm{m}$ (last 9 elements in $y$ direction). The deterministic system has $n=584$ degrees of freedom. A Gaussian hydraulic conductivity $(k)$ with 2D exponential covariance function is considered. The $2 \mathrm{D}$ covariance function is obtained by multiplying a $1 \mathrm{D}$ exponential covariance function depending on $x$, with correlation length $b_{x}=L / 5$; and a $1 \mathrm{D}$ exponential covariance function depending on $y$, with correlation length $b_{y}=W / 5$. Two terms of the KL expansion in each direction are kept, that is, the KL expansion has four matrices for the whole system. The mean value of the hydraulic conductivity is given by $\bar{k}=1 \mathrm{~cm} / \mathrm{s}$, and its standard deviation is $\sigma=0.2 \bar{k}$. The stiffness element matrices are given by

$$
\begin{aligned}
\mathbf{K}_{11_{i j}} & =\int_{0}^{a} \int_{0}^{b} a_{11} \frac{d N_{i}}{d x} \frac{d N_{j}}{d x} \varphi(x, y) d x d y . \\
\mathbf{K}_{22_{i j}} & =\int_{0}^{a} \int_{0}^{b} a_{22} \frac{d N_{i}}{d y} \frac{d N_{j}}{d y} \varphi(x, y) d x d y .
\end{aligned}
$$

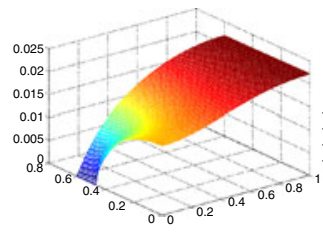

(a) First eigenvector.

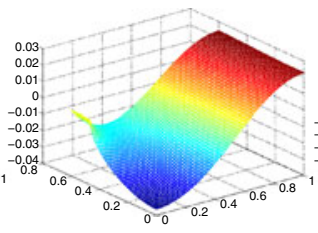

(b) Second eigenvector.

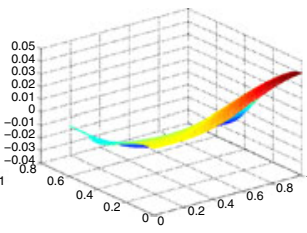

(c) Third eigenvector.

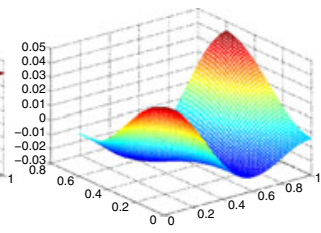

(d) Fourth eigenvector.

Figure 8. First four eigenvectors of the stiffness matrix for the flow through porous media problem. 


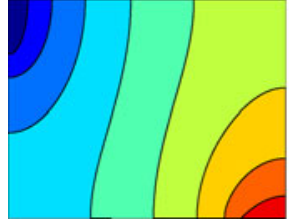

(a) MCS, 10k samples.

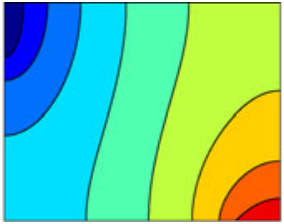

(b) Full PC $(n=584)$.

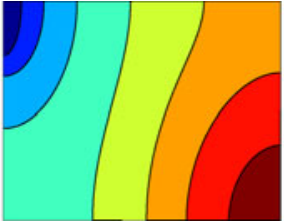

(c) $\operatorname{RPC}(n=5)$.

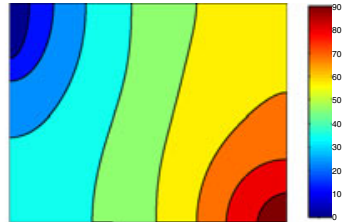

(d) $\operatorname{RPC}(n=20)$.

Figure 9. Contour of the mean of head $(\mathrm{cm})$ obtained with MCS, full PC and reduced systems using the first five and twenty eigenvectors respectively. $x$ and $y$ axis are respectively the positions in $x$ direction with $x \in[-0.5,0.5]$ and $y$ direction with $y \in[-0.3,0.3]$.

The stiffness matrix of the system is given by $\mathbf{K}=\mathbf{K}_{11}+\mathbf{K}_{22}$, where $\mathbf{K}_{11}$ and $\mathbf{K}_{22}$ are obtained by assembling the respective matrices given above. The details of the finite element model can be found, for example, in Reddy (1993). The random variables appearing in the stiffness matrix are $a_{11}=a_{22}=k$. The eigenfunction $\varphi(x, y)=1$ for the deterministic stiffness matrix, and it depends on the covariance function when considering a KL expansion matrix. The polynomial chaos used is of fourth order, so that the total number of polynomials is 70 .

As before, the response is obtained using different methods to compare their performance. MCS is applied using 10000 samples. The size of the linear system to be solved when applying full PC is 40880 . The two reduced systems considered use the first 5 and 20 eigenvectors, respectively. The ratio between the first and the $n$th eigenvalue is shown in figure 7 , and the contours of the first four eigenvectors are shown in figure 8. The two reduced PC system matrices are of sizes 350 and 1400 for the transformed stiffness matrices of sizes 5 and 20, respectively. Contour plots of the mean and standard deviation obtained with the different methods are shown in figures 9 and 10. The contours of the percentage errors in mean and standard deviation between the reduced systems and the full PC are shown in figure 11. The percentage error is calculated with Eq. (46). As before, the accuracy of the response with the proposed method is similar to the one of full PC, but is obtained at a lower cost. This cost is described in table 2, where the CPU time of calculations for the four methods is given. The pdf of the response at coordinate $(x, y)=(0.1714,-0.2857)$ is shown in figure 12 for MCS, full PC and the two reduced order

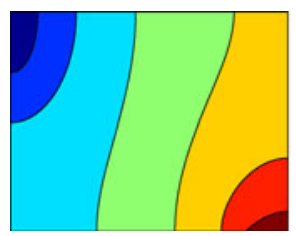

(a) $\mathrm{MCS}, 10 \mathrm{k}$ samples.

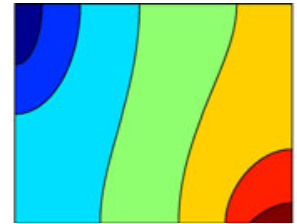

(b) Full PC $(n=584)$.

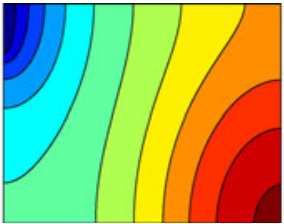

(c) $\operatorname{RPC}(n=5)$.

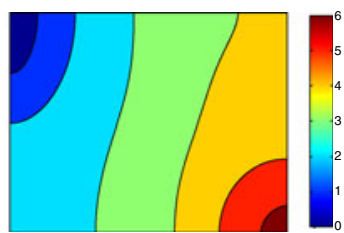

(d) $\operatorname{RPC}(n=20)$.

Figure 10. Contour of the standard deviation of head $(\mathrm{cm})$ obtained with MCS, full PC and reduced systems using the first five and twenty eigenvectors, respectively. $x$ and $y$ axis are respectively the positions in $x$ direction with $x \in[-0.5,0.5]$ and $y$ direction with $y \in[-0.3,0.3]$. 


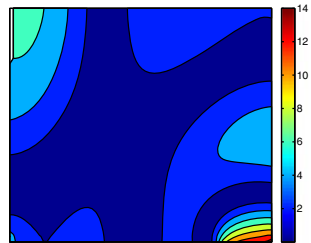

(a) $\varepsilon_{\%}$ of mean $(n=5)$.

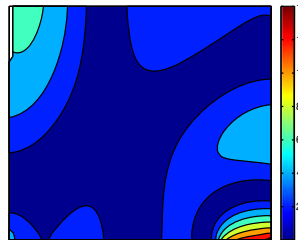

(b) $\varepsilon_{\%}$ of $\sigma$

$(n=5)$.

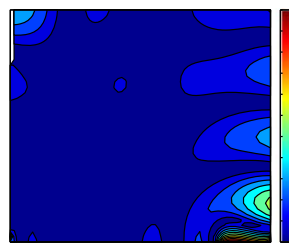

(c) $\varepsilon_{\%}$ of mean $(n=20)$.

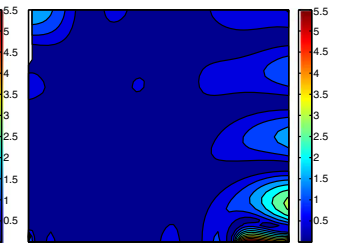

(d) $\varepsilon_{\%}$ of $\sigma$ $(n=20)$.

Figure 11. Contour of percentage error $(\varepsilon \%)$ of mean and standard deviation $(\sigma)$ of RPC (with $n=5$ and $n=20)$ compared to full PC. $x$ and $y$ axis are respectively the positions in $x$ direction with $x \in[-0.5,0.5]$ and $y$ direction with $y \in[-0.3,0.3]$.

Table 2. CPU time (s) of calculations for the full PC and in the proposed reduced method. The cost of calculating complete eigensolutions is $119.7236 \mathrm{~s}$.

\begin{tabular}{lcccc}
\hline & MCS & full PC $(n=584)$ & RPC $(n=5)$ & RPC $(n=20)$ \\
\hline CPU time $(\mathrm{s})$ & $8.3371 \times 10^{3}$ & 192.1206 & 0.8293 & 0.9569 \\
\hline
\end{tabular}

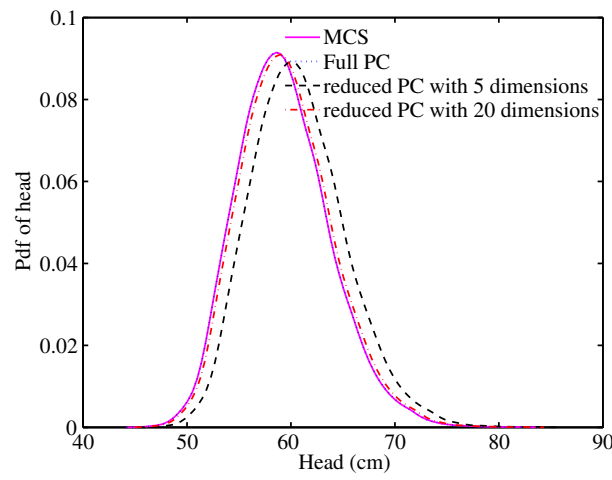

(a) Pdf.

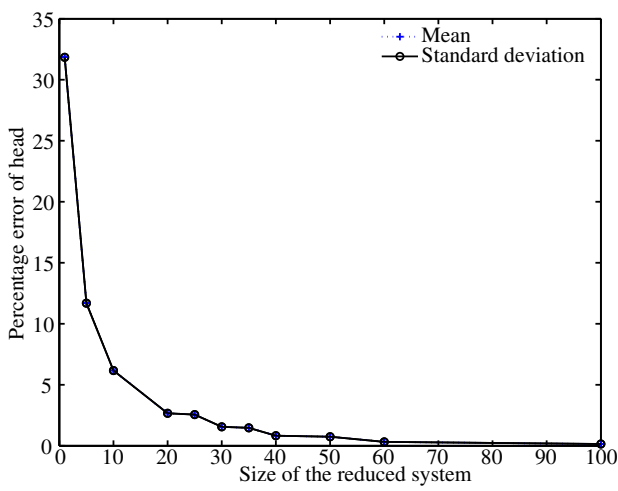

(b) Error of mean and standard deviation.

Figure 12. Pdf of head and percentage errors of mean and standard deviation of head at $(x, y)=$ $(0.1714,-0.2857)$. 


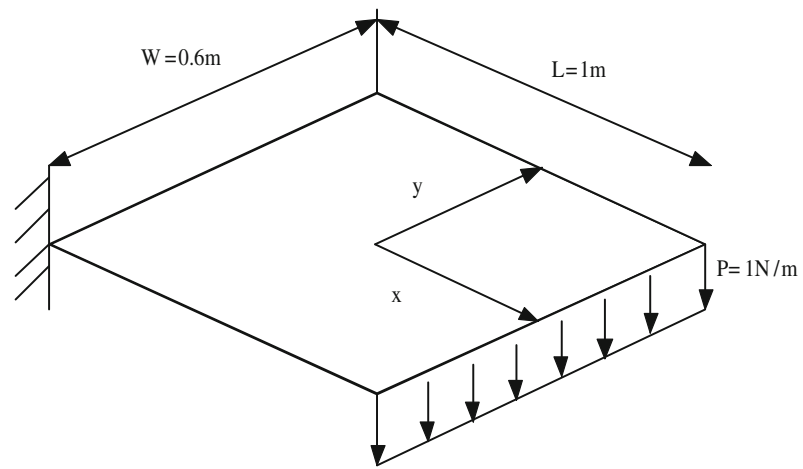

Figure 13. A rectangular elastic plate with stochastic bending rigidity subjected to a line load along one edge.

models. One can observe that with 20 dimensions, the proposed RPC produce results comparable with the full PC with dimension 584. Figure 12b shows that the error compared to the full PC reduces significantly when the size of the reduced system is beyond 50 .

\section{Bending of an elastic plate with stochastic properties}

Numerical example of a plate bending problem is given to investigate the efficiency of the proposed method. The domain is a rectangular plate of length $L=1 \mathrm{~m}$ and width $W=0.6 \mathrm{~m}$, as shown in figure 13. The plate is clamped along its width (coordinate $x=-0.5 \mathrm{~m}$ ) and a uniform distributed load of value $P=1 \mathrm{~N} / \mathrm{m}$ is applied at $x=0.5 \mathrm{~m}$. The plate has 30 elements in $x$ direction and 18 elements in $y$ direction. The deterministic system stiffness matrix is of dimension $n=1710$. The variable $D=\frac{E t^{3}}{12\left(1-\mu^{2}\right)}$ is modelled as a Gaussian random field, where its $2 \mathrm{D}$

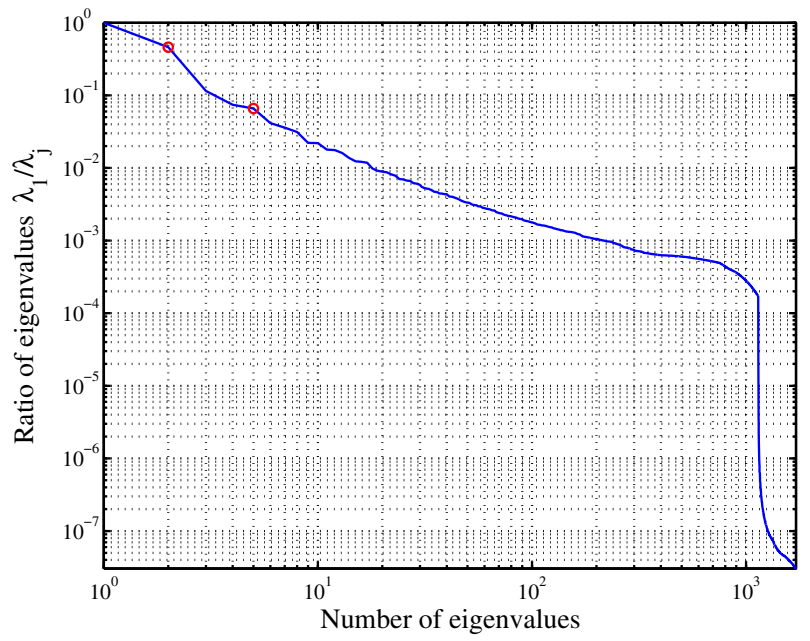

Figure 14. Ratio of eigenvalues for the plate bending problem. 


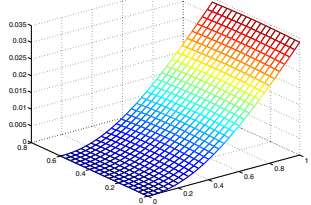

(a) First eigenvector.

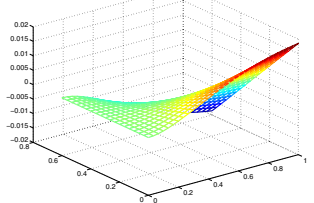

(b) Second eigenvector.

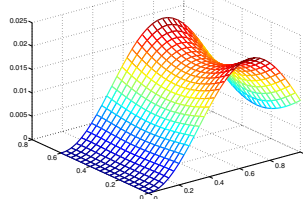

(c) Third eigenvector.

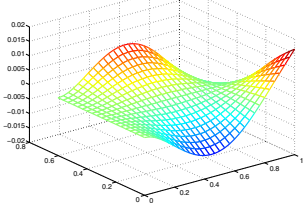

(d) Fourth eigenvector.

Figure 15. First four eigenvectors of the stiffness matrix for the plate bending problem. Only the degrees of freedom corresponding to vertical displacement are represented.

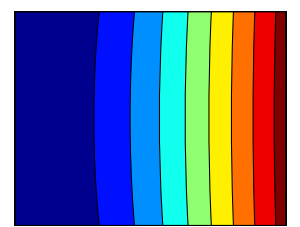

(a) MCS, 10k samples.

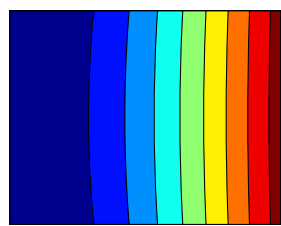

(b) Full PC $(n=1710)$.

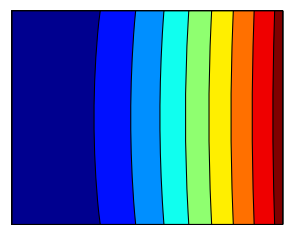

(c) $\operatorname{RPC}(n=2)$.

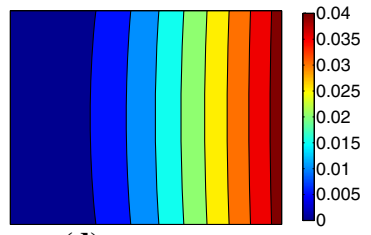

(d) $\operatorname{RPC}(n=5)$.

Figure 16. Contours of the mean of vertical displacement $(\mathrm{m})$ obtained with MCS, full PC and reduced systems using the first two and five eigenvectors respectively. $x$ and $y$ axis are the positions in $x$ direction with $x \in[-0.5,0.5]$ and $y$ direction with $y \in[-0.3,0.3]$.

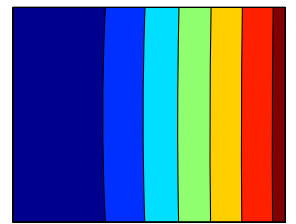

(a) MCS, 10k samples.

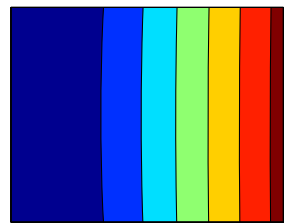

(b) Full PC $(n=1710)$.

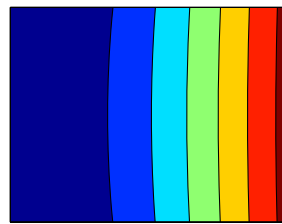

(c) $\operatorname{RPC}(n=2)$

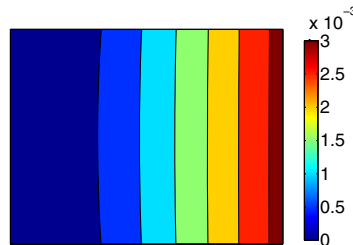

(d) $\operatorname{RPC}(n=5)$

Figure 17. Contours of the standard deviation of vertical displacement (m) obtained with MCS, full PC and reduced PC using the first two and five eigenvectors respectively. $x$ and $y$ axis are respectively the positions in $x$ direction with $x \in[-0.5,0.5]$ and $y$ direction with $y \in[-0.3,0.3]$.

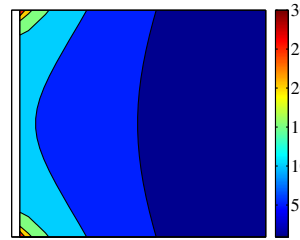

(a) $\varepsilon \%$ of mean $(n=2)$.

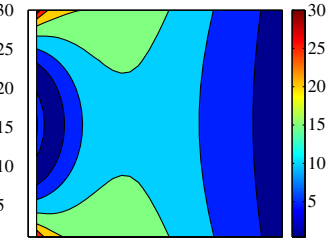

(b) $\varepsilon \%$ of $\sigma(n=2)$.

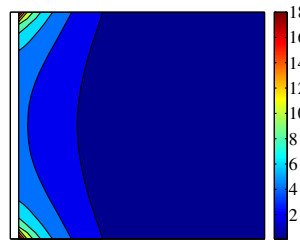

(c) $\varepsilon \%$ of mean $(n=5)$.

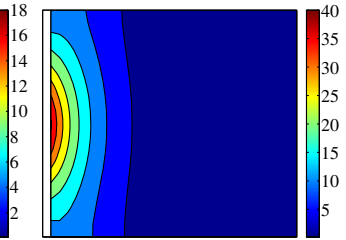

(d) $\varepsilon \%$ of $\sigma(n=5)$.

Figure 18. Contour of percentage error $(\varepsilon \%)$ of mean and standard deviation $(\sigma)$ of vertical displacement. $x$ and $y$ axis are respectively the positions in $x$ direction with $x \in[-0.5,0.5]$ and $y$ direction with $y \in$ $[-0.3,0.3]$. 
Table 3. CPU time (s) of calculations for the full PC and in the proposed reduced method. The cost of calculating complete eigensolutions is $38.0243 \mathrm{~s}$.

\begin{tabular}{ccccc}
\hline & MCS & full PC $(n=1710)$ & RPC $(n=2)$ & RPC $(n=5)$ \\
\hline CPU time & $1.6054 \times 10^{4}$ & 389.2569 & 0.3751 & 0.5074 \\
\hline
\end{tabular}

covariance function is obtained by multiplying an $1 \mathrm{D}$ exponential covariance function depending on $x$, with correlation length $b_{x}=0.2 \mathrm{~m}$; and another 1D exponential covariance function depending on $y$, with correlation length $b_{y}=0.12 \mathrm{~m}$. The mean $\bar{D}$ of $D$ is calculated with $E=200 \mathrm{GPa}, t=3 \mathrm{~mm}$ and $\mu=0.3$, and its standard deviation is $\sigma=0.2 \bar{D}$. Two terms are kept in each 1D KL expansion, therefore, the KL expansion is the sum of a deterministic matrix and four matrices multiplied by independent random variables. Details on the FE method can be found, for example, in Dawe (1984).

The vertical displacement of the system is calculated with four methods: a MCS using 10000 samples, full PC and two reduced systems, where the system is reduced using the two and five first eigenvectors, respectively. The ratio between the first and the $n$th eigenvalue is shown in figure 14. First four eigenvectors for the vertical displacement are shown in figure 15. The two reduced PC system matrices are of sizes $n=140$ and $n=350$ for transformed stiffness matrices of sizes two and five, respectively. Contours of the mean and standard deviation for the four methods are shown respectively in figures 16 and 17 . The percentage error associated with using RPC instead of full PC is shown in figure 18. The percentage error is given by Eq. (46). As before, it is observed that the percentage error between both methods is small, while the computation time is very different, as shown in table 3 . Coordinate $(x, y)=(0.1667,0)$ is chosen to show some features of the method, as the pdf of the vertical displacement at figure 19a. At this position, the percentage errors of mean and standard deviation of the vertical displacement for different reduced systems compared to full PC are shown in figure 19b. One can observe that with 5 dimensions, the proposed RPC produce results comparable with the full PC with dimension 1710. Figure 19b shows that the error compared to the full PC reduces significantly when the size of the reduced system is beyond 20.

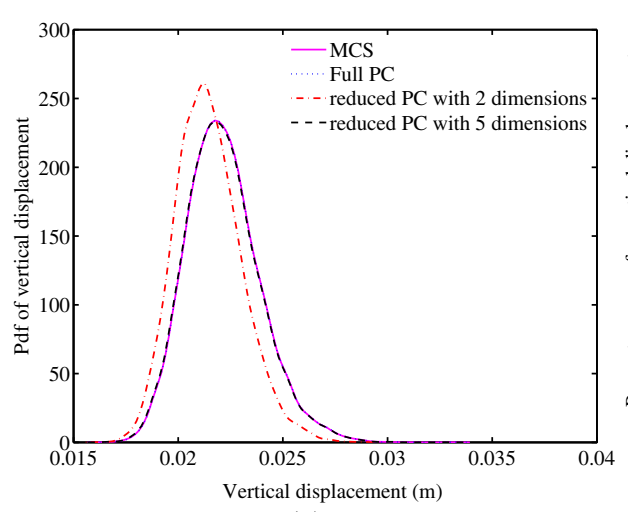

(a) Pdf.

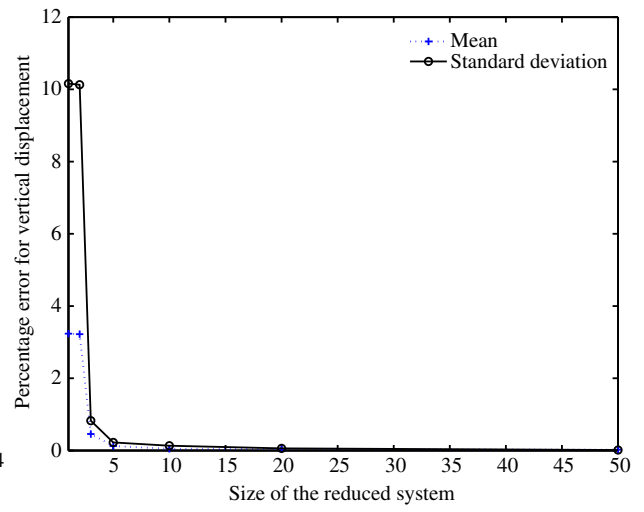

(b) Error of mean and standard deviation.

Figure 19. Pdf and percentage error of mean and standard deviation of vertical displacement, at position $(x, y)=(0.1667,0)$. 


\section{Conclusions}

We consider here the discretized stochastic elliptic partial differential equations. In the classical spectral stochastic finite element approach, each element of the response vector is projected into a basis of polynomials orthogonal with respect to a given pdf, and the associated vectors of coefficients multiplying each polynomial are obtained using a Galerkin type of error minimization approach. Here an alternative approach is investigated. The solution is firstly projected into a finite dimensional orthonormal vector basis, and each element of the associated reduced response vector is then projected into the basis of orthogonal polynomials. As before, the constants arising from the projections are obtained using a Galerkin type of error minimization approach. This error minimization approach is two-fold as both projections, in the vector space and in the functions space, need to be taken into account. The dimension of the linear system to be solved with the classical spectral stochastic finite element approach is obtained by multiplying the number of polynomials used in the projection by the dimension of the system stiffness matrix. In the proposed approach, the dimension of the linear system to be solved is obtained by multiplying the number of polynomials by the number of vectors in the projection basis used. The vectors used in the projection basis are chosen amongst the eigenvectors of the deterministic stiffness matrix, and are the ones corresponding to the $p$ smaller eigenvalues, so that $p$ is the size of the reduced system.

The reduced system can be considered as a preconditioned submatrix of the original system, so that an approximate relation between the errors of the two methods can be derived. The method has been applied to three different systems with random parameters, a bending beam, a flow through porous media with random permeability and a bending plate. For the three systems, a reduction of the system with the method lead to a reduction in computation time with respect to the classical spectral stochastic finite element approach. The accuracy remained similar to that of the original method, in spite of the reduction in the spatial dimension. Keeping the computational cost fixed, the proposed reduced polynomial chaos (RPC) approach allows one to solve a system of larger dimension, or to consider higher order polynomial chaos expansion. Future work will consider coupling RPC with existing stochastic model reduction methods and generalized polynomial chaos.

\section{Acknowledgements}

BP acknowledges the financial support from the Swansea University for the award of a graduate scholarship. SA gratefully acknowledges the support of The Royal Society of London for the Wolfson Research Merit award.

\section{References}

Acharjee S and Zabaras N 2006 A concurrent model reduction approach on spatial and random domains for the solution of stochastic PDEs. Int. J. Numerical Methods in Eng. 12: 1934-1954

Adhikari S 1999 Rates of change of eigenvalues and eigenvectors in damped dynamic systems. AIAA Journal 37: 1452-1458

Adhikari S 2000 Calculation of derivative of complex modes using classical normal modes. Comput. and Struct. 77: 625-633

Adhikari S 2011 Stochastic finite element analysis using a reduced orthonormal vector basis. Computer Methods in Applied Mech. and Eng. 200: 1804-1821 
Adhikari S and Manohar C S 2000 Transient dynamics of stochastically parametered beams. ASCE J. Eng. Mech. 126: 1131-1140

Babuska I, Tempone R and Zouraris G 2005 Solving elliptic boundary value problems with uncertain coefficients by the finite element method: the stochastic formulation. Computer Methods in Applied Mech. and Eng. 194: 1251-1294

Blatman G and Sudret B 2010 An adaptive algorithm to build up sparse polynomial chaos expansions for stochastic finite element analysis. Probabilistic Eng. Mech. 25: 183-197

Charmpis D C, Schueeller G I and Pellissetti M F 2007 The need for linking micromechanics of materials with stochastic finite elements: A challenge for materials science. Computational Materials Sci. 41: 27-37

Dawe D 1984 Matrix and finite element displacement analysis of structures (Oxford, UK: Oxford University Press)

Falsone G and Impollonia N 2002 A new approach for the stochastic analysis of finite element modelled structures with uncertain parameters. Computer Methods in Applied Mech. and Eng. 191: 5067-5085

Feng Y T 2007 Adaptive preconditioning of linear stochastic algebraic systems of equations. Communications in Numerical Methods in Engineering 23: 1023-1034

Foo J and Karniadakis G E 2010 Multi-element probabilistic collocation method in high dimensions. J. Comput. Phys. 229: 1536-1557

Ghanem R and Spanos P 1991 Stochastic finite elements: A spectral approach (New York, USA: SpringerVerlag)

Ghosh D, Ghanem R G and Red-Horse J 2005 Analysis of eigenvalues and modal interaction of stochastic systems. AIAA Journal 43: 2196-2201

Grigoriu M 2006 Galerkin solution for linear stochastic algebraic equations. J. Eng. Mechanics-Asce 132: $1277-1289$

Guedri M, Bouhaddi N and Majed R 2006 Reduction of the stochastic finite element models using a robust dynamic condensation method. J. Sound and Vib. 297: 123-145

Kleiber M and Hien T D 1992 The stochastic finite element method (Chichester: John Wiley)

Lenaerts V, Kerschen G and Golinval J C 2002 Physical interpretation of the proper orthogonal modes using the singular value decomposition. J. Sound and Vib. 249: 849-865

Li C F, Feng Y T and Owen D R J 2006 Explicit solution to the stochastic system of linear algebraic equations $\left(\alpha_{1} A_{1}+\alpha_{2} A_{2}+\cdots+\alpha_{m} A_{m}\right) x=b$. Computer Methods in Applied Mech. and Eng. 195: $6560-6576$

Liu W K, Belytschko T and Mani A 1986 Random field finite-elements. Int. J. Numerical Methods in Eng. 23: $1831-1845$

Ma X and Zabaras N 2009 An adaptive hierarchical sparse grid collocation algorithm for the solution of stochastic differential equations. Journal of Computational Physics 228: 3084-3113

Manohar C S and Adhikari S 1998 Dynamic stiffness of randomly parametered beams. Probabilistic Eng. Mech. 13: 39-51

Matthies H G, Brenner C E, Bucher C G and Soares C G 1997 Uncertainties in probabilistic numerical analysis of structures and solids - Stochastic finite elements. Structural Safety 19: 283-336

Matthies H G and Keese A 2005 Galerkin methods for linear and nonlinear elliptic stochastic partial differential equations. Computer Methods in Applied Mech. and Eng. 194: 1295-1331

Maute K, Weickum G and Eldred M 2009 A reduced-order stochastic finite element approach for design optimization under uncertainty. Structural Safety 31: 450-450

Nair P B and Keane A J 2002 Stochastic reduced basis methods. AIAA Journal 40: 1653-1664

Nouy A 2007 A generalized spectral decomposition technique to solve a class of linear stochastic partial differential equations. Computer Methods in Applied Mech. and Eng. 196: 4521-4537

Nouy A 2008 Generalized spectral decomposition method for solving stochastic finite element equations: Invariant subspace problem and dedicated algorithms. Computer Methods in Applied Mech. and Eng. 197: 4718-4736

Nouy A 2009 Recent developments in spectral stochastic methods for the numerical solution of stochastic partial differential equations. Archives of Computational Methods in Engineering 16: 251-285 
Papoulis A and Pillai S U 2002 Probability, random variables and stochastic processes. Fourth edition (Boston, USA: McGraw-Hill)

Reddy J 1993 An introduction to the finite element models. Second edition (New York, USA: McGraw-Hill) Sachdeva S K, Nair P B and Keane A J 2006 Hybridization of stochastic reduced basis methods with polynomial chaos expansions. Probabilistic Engineering Mechanics 21: 182-192

Sarkar A, Benabbou N and Ghanem R 2009 Domain decomposition of stochastic PDEs: Theoretical formulations. Int. J. Numerical Methods in Eng. 77: 689-701

Stefanou G 2009 The stochastic finite element method: Past, present and future. Computer Methods in Applied Mech. and Eng. 198: 1031-1051

Vanmarcke E H 1983 Random fields (Cambridge Mass.: MIT press)

Wan X L and Karniadakis G E 2006 Beyond Wiener-Askey expansions: Handling arbitrary pdfs. J. Scientific Computing 27: 455-464

Wilkinson J H 1988 The Algebraic Eigenvalue Problem (Oxford, UK: Oxford University Press)

Xiu D B and Karniadakis G E 2002 The Wiener-Askey polynomial chaos for stochastic differential equations. Siam Journal on Scientific Computing 24: 619-644

Yamazaki F, Shinozuka M and Dasgupta G 1988 Neumann expansion for stochastic finite element analysis. J. Eng. Mech.-ASCE 114: 1335-1354

Zhang F 2005 The Schur complement and its applications (New York, USA: Springer Science + Business Media, Inc.) 